\title{
The Standardisation of Terminology of Lower Urinary Tract Function: Pressure-Flow Studies of Voiding, Urethral Resistance and Urethral Obstruction
}

1. Introduction

2. Evaluation of Micturition

2.1. Pressure Flow Studies

2.1. I. Pressure and Flow Rate Parameters

2.1.2. Flow Delay

2.1.3. Presentation of Results

2.2. Urethral Resistance and Bladder Outlet Obstruction

2.2.1. Urethral Function During Voiding

2.2.2. Urethral Resistance

2.2.3. Urethral Activity

2.2.4. Bladder Outlet Obstruction

2.2.5. Methods of Analysing Pressure-Flow Plots

2.3. The Detrusor During Micturition

3. Additional Symbols

References

Appendix: ICS Standard for Digital Exchange of Pressure-Flow Study

A1. Introduction

A2. General Description of Signal Storage

A3. Variahle Yalues and Types

A4. General Structure oiF File and Records

A5. Definitions of Record Types

A6. Signals and Information to be Stored: Minimal Specification and Optional Extensions

A7. Typical File Structure

A8. Aclihowledgements

A9. Addendum: Signal IDs

Neurourol Urodyn (1997) in press

Derek Griffiths (subcommittee chairman), Klaus Hofner, Ron van Mastrigt, Harm Jan Rollema, Anders Spdngber\$ Donald Gleason and Anders Mattiasson (overall chairman)

International Continence Society Subcommittee on Standardisation of Terminology of Pressure-Flow Studies

\section{Introduction}

This report has been produced at the request of the International Continence Society. It was approved at the twenty-fifth annual meeting of the society in Sydney, Australia.

The 1988 version of the collated reports on standardisation of terminology, which appeared in Neurourology and Urodynamics, vol. 7, pp. 403-427, contains material relevant to pressure- 
flow studies in many different sections. This report is a revision and expansion of Sections 4.2 and 4.3 and parts of Sections 6.2 and 7 of the 1988 report. It contains a recommendation for a provisional standard method for defining obstruction on the basis of pressure-flow data.

\section{Evaluation of Micturition}

\subsection{Pressure-Flow Studies}

At present, the best method of analysing voiding function quantitatively is the pressure-flow study of micturition, with simultaneous recording of abdominal, intravesical and detrusor pressures and flow rate (Fig. A.1.6.1).

Direct inspection of the raw pressure and flow data before, during and at the end of micturition is essential, because it allows artefacts and untrustworthy data to be recognised and eliminated. More detailed analyses of pressure-flow relationships, described below, are advisable to aid diagnosis and to quantify data for research studies.

The flow pattern in a pressure-flow study should be representative of free flow studies in the same patient. It is important to eliminate artefacts and unrepresentative studies before applying more detailed analyses.

Pressure-flow studies contain information about the behaviour of the urethra and the behaviour of the detrusor. Section 2.2 deals with the urethra. Detrusor function is considered in Section 2.3 .

\subsubsection{Pressure and Flow Rate Parameters}

Definitions See Fig. A.1.6.1 and Table II; see also Table II Appendix 1, Part 2.

Maximum flow rate is the maximum measured value of the flow rate. Symbol Qmax

Maximum pressure is the maximum value of the pressure measured during a pressure-flow study. Note that this may be attained at a moment when the flow rate is zero. Symbols: $P_{\text {abd, }}$ max, $P_{\text {ves, max }}, P_{\text {det, max }}$.

Pressure at maximum flow is the pressure recorded at maximum measured flow rate. If the same maximum value is attained more than once or if it is sustained for a period of time, then the point of maximum flow is taken to be where the detrusor pressure has its lowest value for this flow rate; abdominal, intravesical and detrusor pressures at maximum flow are all read at this same point. Flow delay (see Section 2.1.2) may have a significant influence and should be considered. Symbols: $P_{\text {abd, Qmax }} P_{\text {ves, Qmax, }} P_{\text {det, }}$ Qmax

Opening pressure is the pressure recorded at the onset of measured flow. Flow delay should be considered. Symbols: $P_{\text {abd, open, }} P_{\text {ves, open, }} P_{\text {det, open }}$. 
Closing pressure is the pressure recorded at the end of measured flow. Flow delay should be considered. Symbols: $P_{\text {abd, cios, }} P_{\text {ves, clos, }} P_{\text {det, clos }}$.

Minimum voiding pressure is the minimum pressure during measurable flow (see Fig. A.1.6.1). It may be, but is not necessarily, equal to the opening pressure or the closing pressure. Example: minimum voiding detrusor pressure, symbol: $P_{\text {det, }}$ min, void.

\subsubsection{Flow Delay}

When a pressure-flow study is performed, the flow rate is measured at a location downstream of the bladder pressure measurement and so the flow rate measurement is delayed. The delay is partly physiological, but it also depends on the equipment. It may depend on the flow rate.

When considering pressure-flow relationships, it may be important to take this delay into account, especially if there are rapid changes in pressure and flow rate. In current practice an average value is estimated by each investigator, from observations of the delay between corresponding pressure and flow rate changes in a number of actual studies. Values from 0.5 to $1.0 \mathrm{~s}$ are typical.

\section{Definition}

Flow delay is the time delay between a change in bladder pressure and the corresponding change in measured flow rate.

\subsubsection{Presentation of Results}

Pressure-flow plots and the nomograms used for analysis should be presented with the flow rate plotted along the $x$-axis and the detrusor pressure along the $y$-axis (see Fig. A.1.6.2). Specify

The value of the flow delay that is used.

\subsection{Urethral Resistance and Bladder Outlet Obstruction}

\subsubsection{Urethral Function During Voiding}

During voiding urethral function may be
(a) normal or
(b) obstructive as a result of
(i) overactivity or
(ii) abnormal structure.

Obstruction due to urethral overactivity occurs when the urethral closure mechanism contracts involuntarily or fails to relax during attempted micturition in spite of an ongoing detrusor contraction. Obstruction due to abnormal structure has an anatomical basis, e.g., urethral stricture or prostatic enlargement. 


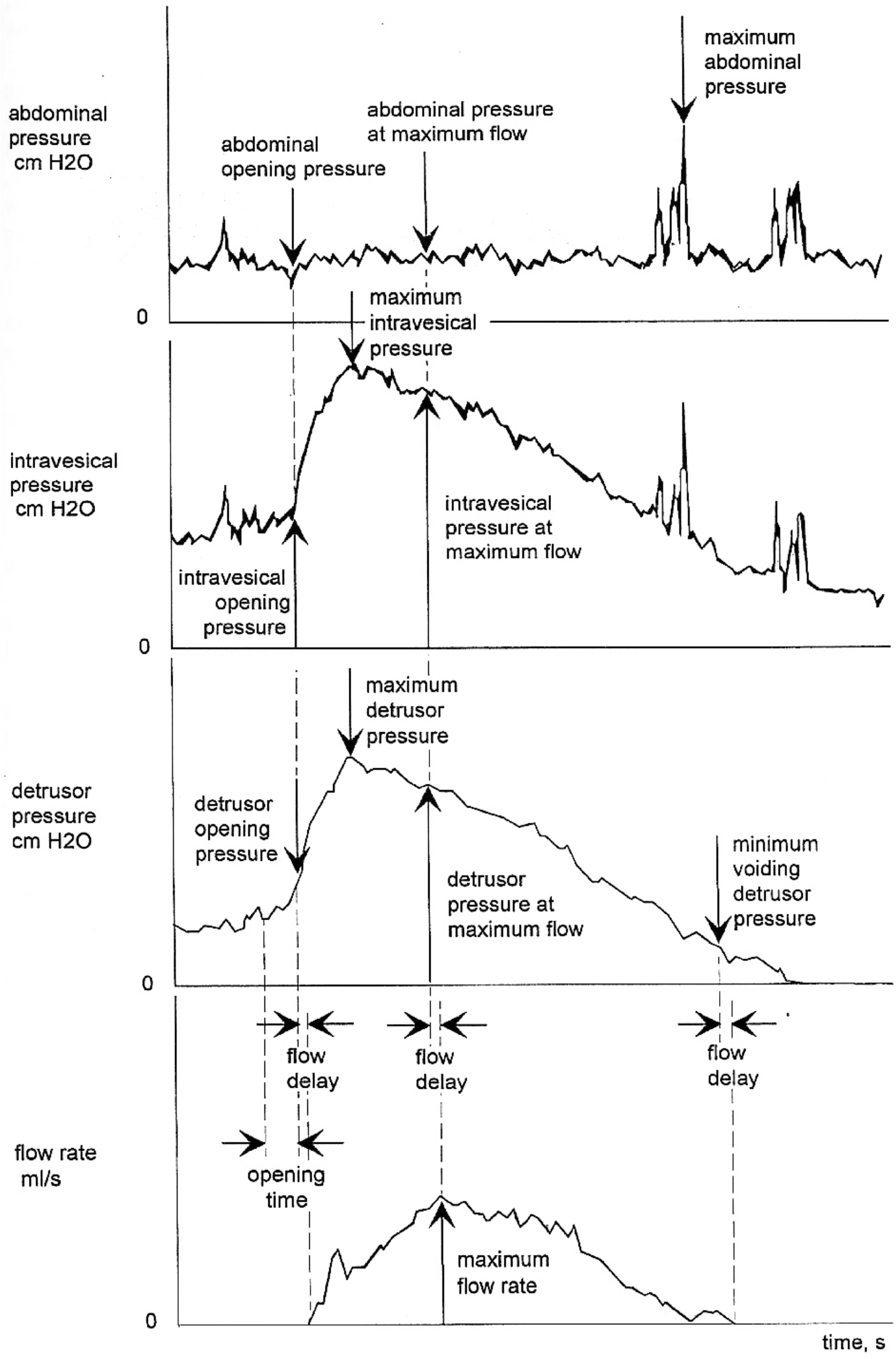

Fig. A.1.6.1 Diagram of a pressure-flow study with nomenclature recommended in this report. 


\subsubsection{Urethral Resistance}

Urethral resistance is represented by a relation between pressure and flow rate, describing the pressure required to propel any given flow rate through the urethra. The relation is called the urethral resistance relation (URR).

An indication of the urethral resistance relation is obtained by plotting detrusor pressure against flow rate. The most accurate procedure, which requires a computer or an $x / y$ recorder, is a quasicontinuous plot showing many pairs of corresponding pressure and flow rate values (Fig. A.1.6.2). A simpler procedure, which can be performed by hand, is to plot only two or three pressure-flow points connected by straight lines; for example, the points of minimum voiding pressure and of maximum flow may be selected. In whatever way the plot is made, flow delay should be considered.

A further simplification is to plot just one point showing the maximum flow rate and the detrusor pressure at maximum flow. Flow delay should be considered.

Methods of analysing pressure-flow plots are further discussed below.

\subsubsection{Urethral Activity}

Ideally, the urethra is fully relaxed during voiding. The urethral resistance is then at its lowest and the detrusor pressure has its lowest value for any given flow rate. Under these circumstances the urethral resistance relation is defined by the inherent mechanical and morphological properties of the urethra and is called the passive urethral resistance relation (Fig. A.1.6.2).

Urethral activity can only increase the detrusor pressure above the value defined by the passive urethral resistance relation. Therefore, any deviations of the pressure-flow plot from the passive urethral resistance relation toward higher pressures are regarded as due to activity of the urethral or periurethral muscles, striated or smooth.

\subsubsection{Bladder Outlet Obstruction}

Obstruction is a physical concept which is assessed from measurements of pressure and flow rate, made during voiding. Whether due to urethral overactivity or to abnormal structure, obstruction implies that the urethral resistance to flow is abnormally elevated. Because of natural variation from subject to subject, there cannot be a sharp boundary between normal and abnormal. Therefore the definition of abnormality requires further elaboration.

\subsubsection{Methods of Analysing Pressure-Flow Plots}

The results of pressure-flow studies may be used for various purposes, for example for objective diagnosis of urethral obstruction or for statistical testing of differences in urethral resistance between groups of patients. For these purposes methods have been developed to 
quantify pressure-flow plots in terms of one or more numerical parameters. The parameters are based on aspects such as the position, slope or curvature of the plot. Some of these methods are primarily intended for use in adult males with possible prostatic hypertrophy.

\begin{tabular}{|l|l|l|l|l|l|}
\hline Method & Aim & $\begin{array}{l}\text { Number of } \\
p / Q \text { points }\end{array}$ & $\begin{array}{l}\text { Assumed } \\
\text { shape of } \\
\text { URR }\end{array}$ & $\begin{array}{l}\text { Number of } \\
\text { parameters }\end{array}$ & $\begin{array}{l}\text { Number of } \\
\text { classes or } \\
\text { continuous }\end{array}$ \\
\hline $\begin{array}{l}\text { Abrams- } \\
\text { Griffiths } \\
\text { nomogram } 1\end{array}$ & diagnosis & 1 & n/a & $n / a$ & 3 \\
\hline $\begin{array}{l}\text { Spangberg } \\
\text { nomogram }\end{array}$ & diagnosis & 1 & n/a & n/a & 3 \\
\hline $\begin{array}{l}\text { URA }^{3}, 4 \\
\text { linPURR }\end{array}$ & resistance & 1 & curved & 1 & continuous \\
\hline $\begin{array}{l}\text { Schafer } \\
\text { PURR }\end{array}$ & resistance & $1 a$ & linear & 1 & 7 \\
\hline CHESS $^{7}$ & resistance & many & curved & 2 & continuous \\
\hline OBI ${ }^{8}$ & resistance & many & linear & 1 & continuous \\
\hline $\begin{array}{l}\text { Spangberg et } \\
\text { al. }\end{array}$ & resistance & many & $\begin{array}{l}\text { linear or } \\
\text { curved }\end{array}$ & 3 & $\begin{array}{l}\text { continuous }+ \\
\text { categories }\end{array}$ \\
\hline DAMPF 9 & resistance & 2 & linear & 1 & continuous \\
\hline $\begin{array}{l}\text { A/G } \\
\text { number } 10\end{array}$ & resistance & 1 & linear & 1 & continuous \\
\hline
\end{tabular}

a Schäfer uses 2 points to draw a linear relation but the point at maximum flow determines the resistance grade.

Some methods of analysis are shown in Table I.

Quantification of Urethral Resistance In all current methods, urethral resistance is derived from the relationship between pressure and flow rate. A commonly used method of demonstrating this relationship is the pressure-flow plot. The lower pressure part of this plot is taken to represent the passive urethral resistance relation (see Fig. A.1.6.2). In general, the higher is the pressure for a given flow rate, and/or the steeper or more sharply curved upward is this part of the plot, the higher is the urethral resistance. The various methods differ in how the position, slope, and/or curvature of the plot are quantified and how and whether they are combined. Some methods grade urethral resistance on a continuous scale; others grade it in a small number of classes (Table I). If there are few classes, small changes in resistance may not be detected. Conversely, a small change on a continuous scale may not be clinically relevant.

Some methods result in a single parameter; others result in two or more parameters (Table I). A single parameter makes it easy to compare different measurements. A larger number of 
parameters makes comparison more difficult but potentially gives higher accuracy and validity. If there are too many parameters, however, accuracy may be compromised by poor reproducibility.

Choice of Method Some methods in Table I are intended primarily to quantify urethral resistance. Others are intended only for the diagnosis of obstruction. Methods that quantify urethral resistance on a scale can also be used to aid diagnosis of obstruction by comparison with cutoff values. In every case an equivocal zone may be included.

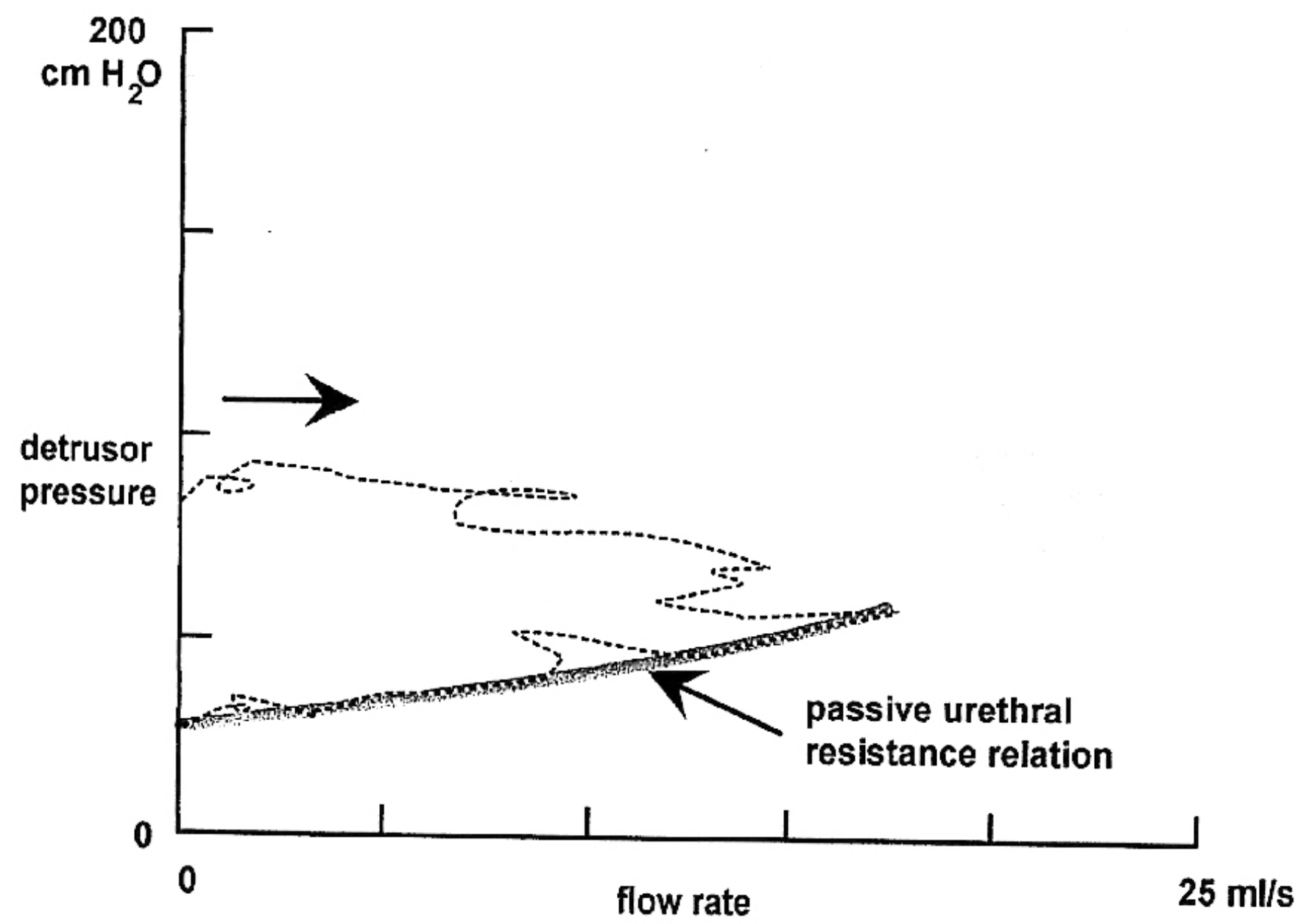

Fig. A.1.6.2 Plot of detrusor pressure against flow rate during voiding (broken curve), providing an indication of the urethral resistance relation (URR). The continuous smooth cune is an estimate of the passive urethral resistance relation.

Because of their underlying similarity, all the above methods classify clearly obstructed and clearly unobstructed pressure-flow studies consistently, but there is some lack of agreement in a minority of cases with intermediate urethral resistance. 
Any of the methods of analysing pressure-flow studies may be useful for a particular purpose. In selecting a method, investigators should consider carefully what their aims are and which method is best suited to attain them.

Identification of Optimum Methods For a subsequent report the International Continence Society will compare the above methods with each other and may also develop new methods, with the aim of reaching a consensus on their use. The Society will continue to seek better ways of clinically validating these methods. The following procedure has been agreed on.

Making use of good-quality data stored in digital format, the following databases will be examined:

1. Pressure-flow studies in untreated men with lower urinary tract symptoms and signs suggestive of benign prostatic obstruction.

2. Pressure-flow studies repeated after a time interval with no intervention. 3. Pressure-flow studies before and after TURP.

4. Pressure-flow studies before and after alternative therapeutical intervention that causes a small change in urethral resistance.

Database 1 will be used to determine which existing or new methods adequately describe the actual pressure-flow plots of male patients with lower urinary tract symptoms. Database 2 will be used to determine the reproducibility of the various methods. Database 3 will be used to determine in which groups of patients TURP significantly reduces urethral resistance, and hence which patients are indeed obstructed. Database 4 will be used to test the sensitivity of the various methods to small changes of urethral resistance.

On the basis of these analyses, the International Continence Society will attempt to identify:

(i) A simple and reproducible method with high validity of diagnosing obstruction.

(ii) A sensitive and reproducible method with high validity of measuring urethral resistance and changes in resistance.

Provisional Recommendation Pending the results of these procedures, it is recommended that investigators reporting pressure-flow studies in adult males, particularly those with benign prostatic hyperplasia, use one simple standard method of analysis in addition to any other method that they have selected, so that results from different centres can be compared. For this provisional method it is recommended that urethral resistance is specified by the maximum flow rate and the detrusor pressure at maximum flow, i.e., by the pair of values $\left(Q_{\max }, P_{\text {det, }}\right.$ Qmax). A provisional diagnostic classification may be derived from these values as follows:

- If $\left(P_{\text {det, }} Q \max -2 Q_{\max }\right)>40$ the pressure-flow study is obstructed.

- If $\left(P_{\text {det, }} Q \max -2 Q_{\max }\right)<20$ the pressure-flow study is unobstructed.

- Otherwise the study is equivocal. 
In these formulae pressure and flow rate are expressed in $\mathrm{cmH}_{2} \mathrm{O}$ and $\mathrm{ml} / \mathrm{s}$ respectively. This method is illustrated graphically in Fig. A.1.6.3. It may be referred to as the provisional ICS method for definition of obstruction.

The equivocal zone of the provisional method (Fig. A.1.6.3) is similar but not identical to those of the Abrams-Griffiths and Spangberg nomograms and to the region defining linPURR grade II. For micturitions with low to moderate flow rates it is consistent with cut off values used to define obstruction in the URA and CHESS methods.

\subsection{The Detrusor During Micturition}

During micturition the detrusor may be
(a) Acontractile
(b) Underactive
(c) Normal

(a) The acontractile detrusor is one that cannot be demonstrated to contract during urodynamic studies.

(b) Detrusor underactivity is defined as a detrusor contraction of inadequate magnitude and/or duration to effect complete bladder emptying in the absence of urethral obstruction. (Concerning the elderly see (c). Both magnitude and duration should be considered in the evaluation of detrusor contractility. 


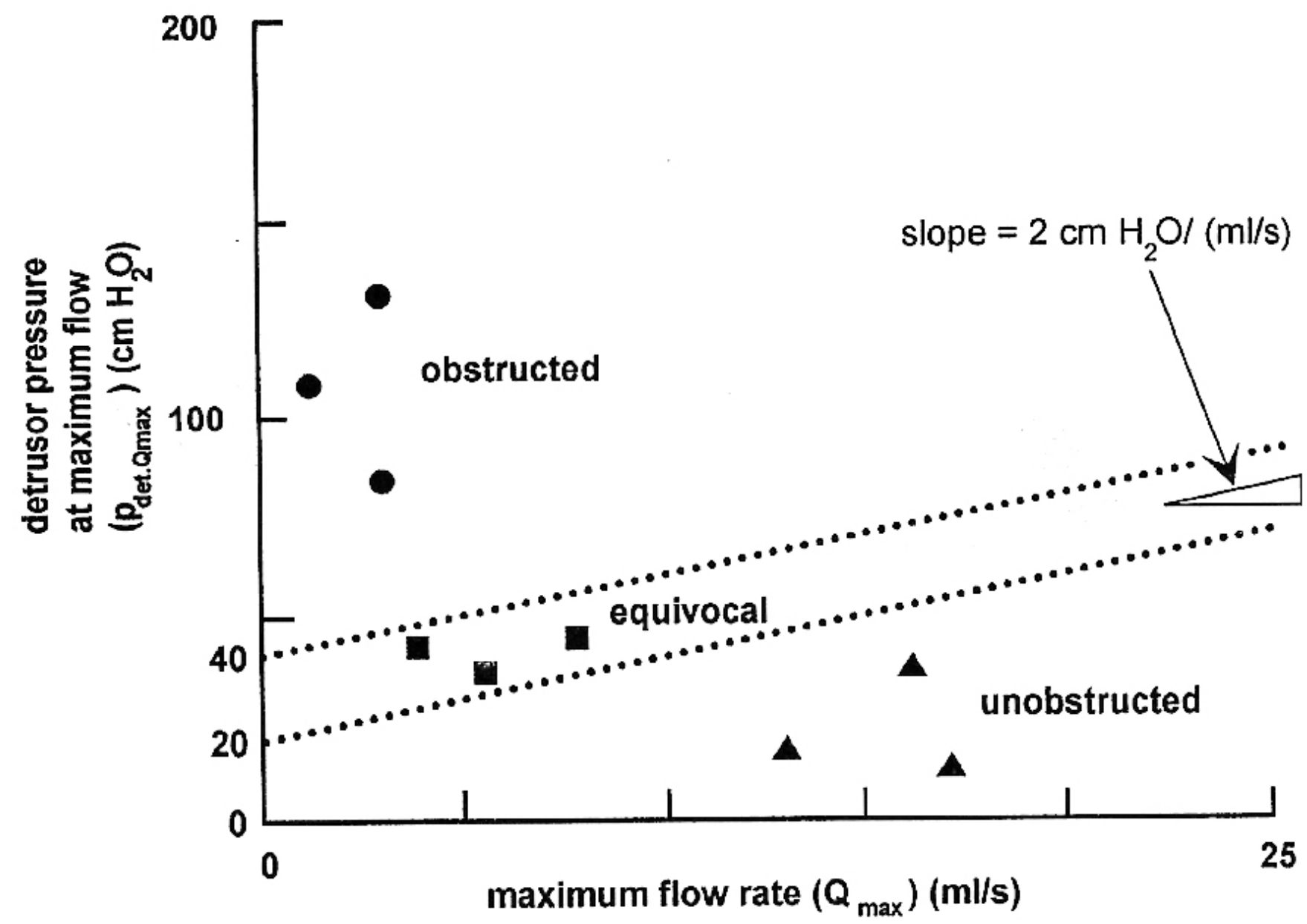

Fig. A.1.6.3 Provisional ICS method for definition of obstruction. The points represent schematicaHy the values of maximum flow rate and detrusor pressure at maximum flow for 9 different voids, 3 in each class.

(c) Normal detrusor contractility. In the absence of obstruction, a normal detrusor contraction will effect complete bladder emptying. Detrusor contractility in the elderly may need special consideration.

For a given detrusor contraction, the magnitude of the recorded pressure rise will depend on the outlet resistance. In general, the higher the detrusor pressure and/or the higher the flow rate, the stronger is the detrusor contraction. The magnitude of the detrusor contraction may be approximately quantified by means of a nomogram applied to the pressure-flow plot or by calculation.

\section{Additional Symbols}

Qualifiers that can be used to form symbols for variables relevant to voiding are shown in Table II. These are additions to those in Table II of the 1988 standardisation report. 
Table II. Qualifiers that can be used to indicate pressure and flow variables relevant to voiding

Qualifiers

At maximum flow

During voiding

Opening

Closing

Examples

$P_{\text {det, }}$ Qmax

$P_{\text {det min, void }}$

$P_{\text {ves, open }}$

$P_{\text {ves. clos }}$
$Q_{\max }$

void

open -!

clos

Detrusor pressure at maximum flow

Minimum voiding detrusor pressure

Intravesical opening pressure

Intravesical closing pressure

When possible, qualifiers should be printed as subscripts (see above). Note that the preferred symbol for pressure is lower-case $p$, while the symbol for flow rate is capital (upper-case) $Q$.

\section{References}

1. Abrams PH, Griffiths DJ (1979). The assessment of prostatic obstruction from urodynamic measurements and from residual urine. Br J Urol 51:129-134.

2. Spangberg A, Terio H, Ask P, Engberg A (1991). Pressure/flow studies preoperatively and postoperatively in patients with benign prostatic hypertrophy: estimation of the urethral pressure/ flow relation and urethral elasticity. Neurourol Urodyn 10:139-167.

3. Griffiths D, Van Mastrigt R, Bosch R (1989). Quantification of urethral resistance and bladder function during voiding, with special reference to the effects of prostate size reduction on urethral obstruction due to benign prostatic hypertrophy. Neurourol Urodyn 8:17-27.

4. Rollema HJ, van Mastrigt R (1992). Improved indication and follow-up in transurethral resection ofthe prostate (TUR) using the computer program CLIM. J Urol 148:111-116.

5. Schafer W (1990). Basic principles and clinical application of advance analysis of bladder voiding function. Urol Clin N Am 17:553-566.

6. Schafer W (1983). The contribution of the bladder outlet to the relation between pressure and flow rate during micturition. In: Hinman F Jr (ed) Benign prostatic hypertrophy. New York: SpringerVerlag, pp.470-496.

7. Hofner K, Kramer AEJL, Tan HK, Krah H, Jonas U (1995). CHESS classification of bladder outflow obstruction. A consequence in the discussion of current concepts. World J Urol 13:59-64.

8. Kranse M, Van Mastrigt R (1991). The derivation of an obstruction index from a three parameter model fitted to the lowest part of the pressure flow plot. J Urol 145:261A.

9. Schafer W (1995). Analysis of bladder-outlet function with the linearized passive urethral resistance relation, linPURR, and a disease-specific approach for grading obstruction: from complex to simple. World J Urol 13:47-58.

10. Lim CS, Abrams P (1995). The Abrams-Griffiths nomogram. World J Urol 13:34-39. 


\section{ICS Standard for Digital Exchange of Pressure-Flow Study Data}

\section{A1. Introduction}

To facilitate exchange of digital urodynamic data a standard file format is required. In this document an ICS standard is summarised. Its primary purpose is to enable data from pressure-flow studies to be exchanged. Enough detail is given to allow exchange of other urodynamic data. Extensions may be made as described in Section A5.

\section{A2. General Description of Signal Storage}

For each pressure-flow study, urodynamic signals sampled equidistantly with an A/D converter, and other associated information, are stored in one binary MS-DOS compatible file on a 5.25 or 3.5 inch floppy disk. The stored signals start 10 seconds before a detectable change in the flow rate signal and continue until 10 seconds after the flow rate has finally returned to baseline. Whenever possible, all signals should be stored at the same sample rate. In this case all signals have the same length, i.e., contain the same number of bytes.

The file name and extension are A B C DEF G H. ICS, where A B C DEF G H stands for a unique measurement identification string. In the case of a multicentre study requiring exchange of data, "unique" implies that this string is defined by the coordinating centre. In the ICS-BPH study for instance this would be a number consisting of (first) 3 digits for centre number, (next) 3 digits for patient number and (finally) 2 digits for identification of successive measurements made on this one patient in one or more sessions.

The file consists of a number of records of various types. In the ICSMFF proposal on which this document is partly based (see Section A8: Acknowledgements) 14 different types of record are defined, numbered -1 and 1 to 13 . Existing record types may not be modified but extensions may be implemented by defining new types of record. For ICS purposes 6 additional types of record, types 14 to 19, are required and are defined in Section A5. In addition, the structures of records of types 8 and 9 are further elaborated in Section A5. 


\section{A3. Variable Values and Types}

In this report actual values for bytes or words may be given in hexadecimal notation as follows: Hex:DD for 8-bit bytes, Hex:DDDD for 16-bit words, and so on, where D stands for a hexadecimal digit.

The following variable types are used in the definitions in the following sections:

$\begin{array}{ll}\text { byte } & \text { unsigned 8-bit value } \\ \text { word } & \text { unsigned 16-bit value } \\ \text { integer } & \text { signed 16-bit value } \\ \text { dword } & \text { unsigned 32-bit value } \\ \text { string }[N] & N \text { bytes including a terminating Hex:OO } \\ \text { word }[N] & N \text { words }\end{array}$

\section{A4. General Structure of File and Records}

Each file A B C DE F G H . ICS consists of a number of records. The number of records is not predefined. Records may be of different types, containing different kinds of data. For example a record may contain a description of a urodynamic signal or information about the patient. 


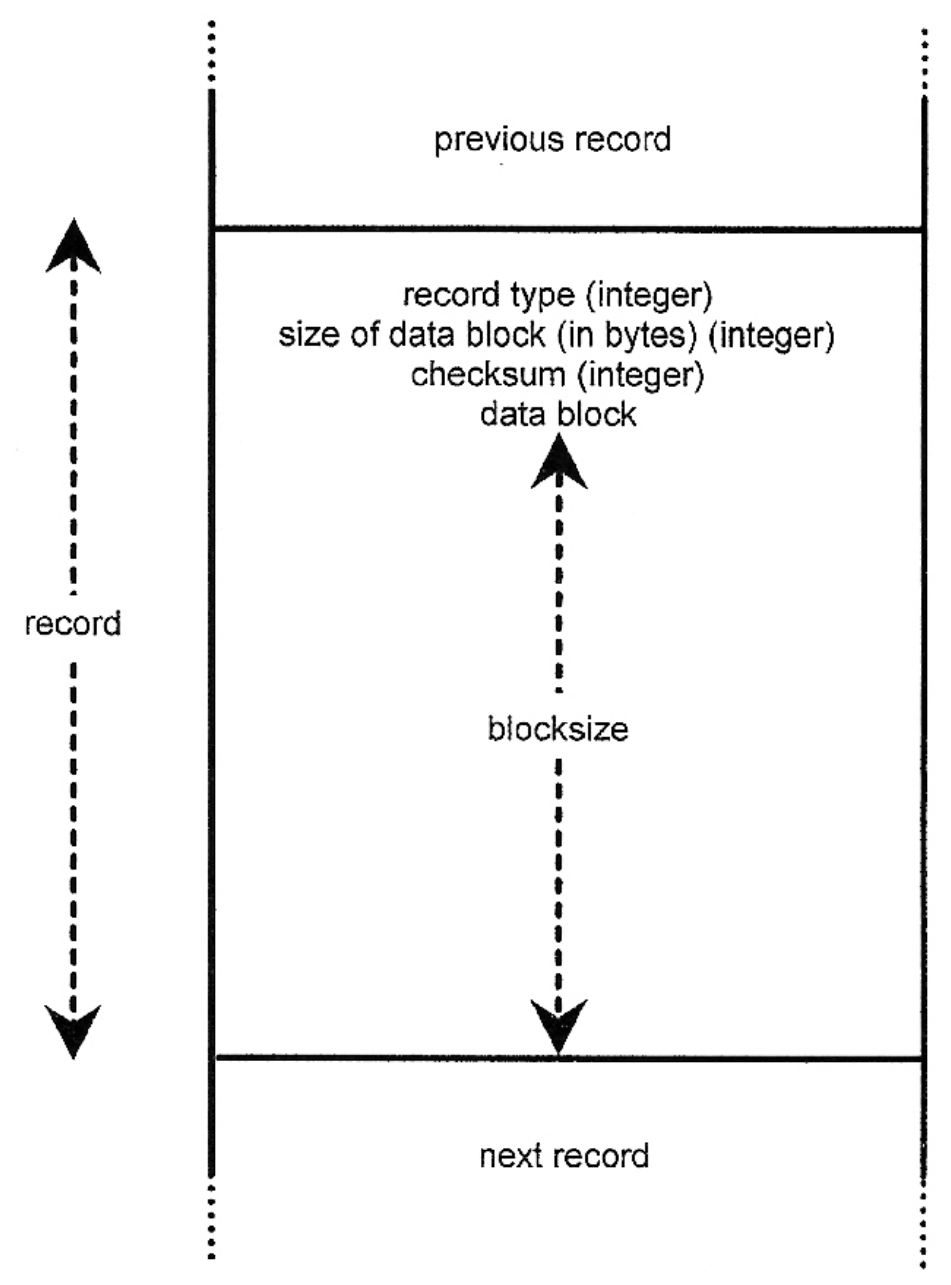

Fig. A.1.6.A1 Schematic structure of file and records.

Each record starts with a descriptor containing the record type, the size of the data block in bytes and a checksum. The descriptor is followed by the block containing the actual data (see Fig. A.1.6.A1).

The record type and the block size are integers. The checksum is an integer such that the 16bit sum of the record type, the data block size and the checksum is zero.

\section{A5. Definitions of Record Types}

The record type defines what kind of data is contained by the data block in the record. The only constraint is that the record type is unique.

Backward compatibility is preserved by the requirement that previously defined record types may not be modified. Extensions are implemented by defining new types of record. New types of signal may be implemented by defining new signal IDs, together with signal names and units. Forward compatibility will be insured if the user does not try to handle unknown types of record or unknown signal IDs. 
A register of the types of record in use, and a register of signal IDs, signal names and units, will be administered centrally by the ICS. This standard contains initial versions of the registers. Proposed extensions should be communicated to the ICS for registration.

\section{Default Values}

Empty fields are not permitted. Occasionally, the value for a certain field may not be available. In this case a default value should be stored (see below).

Signal ID

See Section A9: Addendum.

The following sections describe the types of record needed to implement this ICS standard. Other types are described in the document ICS Measure File Format (ICSMFF) version 1.00, dated 14.10.93 (see Section A8: Acknowledgements).

Record Type 8: The Measurement Marker Record

This type of record defines a marker within the measurement and an associated comment.

$\begin{array}{lll}\text { Record type } & 8 \quad \text { integer } & \\ \text { Blocksize } & 1+N+1+4+M & \text { integer } \\ \text { Checksum } & -(8+\text { blocksize }) & \text { integer } \\ \text { Data block } & \text { marker type } & \text { byte } \\ & \text { name } & \text { string }[N] \\ & \text { signal ID } & \text { byte } \\ & \text { position } & \text { dword } \\ & \text { comment } & \text { string }[M]\end{array}$

The following information should be stored in these fields:

In the field:

$\begin{array}{ll}\begin{array}{l}\text { marker type } \\ \text { name }\end{array} & \begin{array}{r}0 \text { default value } \\ \text { (other types are defined in the ICSMFF document) } \\ \text { empty string as default value } \\ \text { (empty string contains only the terminating Hex:00, so } \\ \text { that } \mathrm{N}=1 \text { ) }\end{array} \\ \begin{array}{l}\text { signal ID } \\ \text { position }\end{array} & \begin{array}{l}\text { as in Section A9: Addendum } \\ \text { the number of the sample (16-bit word) with which the marker and } \\ \text { comment are associated }\end{array} \\ \text { comment } & \begin{array}{l}\text { the comment string, terminated with Hex:OO } \\ \text { the }\end{array}\end{array}$

Record Type 9: The Measurement Comment Record 
This record stores a comment. For the ICS standard it should be used to identify the patient and the type of the measurement.

$\begin{array}{lll}\text { Record type } & 9 & \text { integer } \\ \text { Blocksize } & N & \text { integer } \\ \text { Checksum } & -(9+\text { blocksize }) & \text { integer } \\ \text { Data block } & \text { measurement comment } & \text { string }[N]\end{array}$

The following information should be stored:

In the field:

measurement comment a free format string, terminated with Hex:OO, that uniquely identifies patient and measurement for the originating centre. This string would not only include a patient ID, e.g. from the local hospital information system, but also the relation of this measurement to a particular research study, e.g. "post-TURP investigation". For use in a specific multi-centre study, this string may be specified more strictly.

Record Type 14: The ICS Signal Property Record

This record type describes the properties of one stored signal, including its name, the unit of measurement, the zero and full scale values, and the sample rate (which if possible should be identical for all signals).

$\begin{array}{lll}\text { Record type } & 14 & \text { integer } \\ \text { Blocksize } & 1+\mathrm{N}+\mathrm{M}+2+4 & \text { integer } \\ \text { Checksum } & -(14+\text { blocksize }) & \text { integer } \\ \text { Data block } & \text { signal id } & \text { byte } \\ & \text { signal name } & \text { string }[N] \\ & \text { unit } & \text { string }[M] \\ & \text { binzero } & \text { integer } \\ & \text { binsize } & \text { integer } \\ & \text { fullscale } & \text { integer } \\ & \text { sample rate } & \text { dword }\end{array}$

The following information should be stored in these fields: In the field:

signal ID as specified in Section A9: Addendum

signal name as specified in Section A9: Addendum

unit as specified in Section A9: Addendum

binzero the signal value represented in the data samples field of record type 18 by the word 0

binsize the full scale binary sample value

fullscale the signal value represented in the data samples field of record type 18 by the full scale binary value 
sample rate

sample rate specification in samples/s $(\mathrm{Hz})$. The sample rate is in 16.16 format: i.e., it is a long integer, where bits 16 through 31 specify the integer part of a fixed point number and bits 0 through 15 specify the decimal part.

The following values are recommended:

- Sample rate at least 10 samples/s.

- Pressure signals bipolar up to $+200 \mathrm{cmH} 2 \mathrm{O}$ with a minimum resolution of $0.5 \mathrm{cmH} 2 \mathrm{O} / \mathrm{bit}$.

- Flow rate signal unipolar up to $50 \mathrm{ml} / \mathrm{s}$ with a minimum resolution of $0.05 \mathrm{ml} / \mathrm{s} / \mathrm{bit}$.

Example showing how sample rate is to be specified:

Suppose the rate of sampling is $512.5 \mathrm{~Hz}$. Then the value to be stored in the field sample rate is the 16.16 value Hex:02008000.

Example showing how binzero, binsize and fullscale are to be used:

Suppose the abdominal pressure is sampled bipolarly with a ten bit A/D converter. Then the binary value will range from $O$ to 1023 . Suppose the $A / D$ converter is configured so that this range represents the pressure range from -50 to $200 \mathrm{cmH} 2 \mathrm{O}$, then the three values to be stored are:

binzero -50

binsize 1023

fullscale 200

Record Type 15: The ICS Patient Data Record

This record type contains the basic demographic data of the patient. It contains the actual text and numerical fields plus an index table to facilitate location of a particular field. The index gives the relative position within the data block.

$\begin{array}{lll}\text { Record type } & 15 & \text { integer } \\ \text { Blocksize } & 17^{\star} 2+N+M+O+P+Q+R+S+ & \\ & T+U+V+W+X+Y+3+2 & \text { integer } \\ \text { Checksum } & -(15+\text { blocksize }) & \text { integer } \\ \text { Data block } & \text { surname index } & \text { word } \\ & \text { first name index } & \text { word } \\ & \text { maiden name index } & \text { word } \\ & \text { ID index } & \text { word } \\ & \text { street index } & \text { word } \\ & \text { housenumber index } & \text { word } \\ & \text { city index } & \text { word } \\ & \text { country index } & \text { word } \\ & \text { postcode index } & \text { word } \\ & \text { phone index } & \text { word } \\ & \text { height index } & \text { word }\end{array}$




$\begin{array}{lc}\text { weight index } & \text { word } \\ \text { sex index } & \text { word } \\ \text { comments index } & \text { word } \\ \text { birth day index } & \text { word } \\ \text { birth month index } & \text { word } \\ \text { birth year index } & \text { word } \\ \text { surname } & \text { string [N] } \\ \text { first name } & \text { string [M] } \\ \text { maiden name } & \text { string [O] } \\ \text { ID } & \text { string [P] } \\ \text { street } & \text { string [Q] } \\ \text { housenumber } & \text { string [R] } \\ \text { city } & \text { string [S] } \\ \text { country } & \text { string [T] } \\ \text { postcode } & \text { string [U] } \\ \text { phone } & \text { string [V] } \\ \text { height } & \text { string [W] } \\ \text { weight } & \text { string [X] } \\ \text { comments } & \\ \text { sex } & \text { string [Y] } \\ \text { birth day } & \text { byte* } \\ \text { birth month } & \text { byte } \\ \text { birth year } & \text { byte } \\ \text { wordt } & \end{array}$

${ }^{*} 0=$ male, 1 = female.

t Birth year is expressed in full, e.g., 1895, 1995, 2005.

The first name field may contain a middle or other initial in addition to the first name.

As a default, an empty string (containing only flex:OO) may be stored in any of the string fields for which information is not available or may not be disclosed because of ethical considerations. In this case the length ( $N, M$, etc.) of the string $\sim s 1$.

The index fields contain the position (in bytes) of the corresponding text or numerical field, relative to the start of the data block. Thus the value to be stored in surname index is $17^{*} 2$; the value to be stored in f rst name index is $17^{*} 2+N$; and so on. All the index fields must be completed.

\section{Record Type 16: The ICS Source Record}

This record type specifies the origin of the measurement. It consists of an index table together with the actual text fields.

Record type Blocksize
16 $10^{*} 2+\mathrm{N}+\mathrm{M}+\mathrm{O}+\mathrm{P}+\mathrm{Q}+\mathrm{R}+\mathrm{S}+$ $\mathrm{T}+\mathrm{U}+\mathrm{V}$ integer

integer 


$\begin{array}{lll}\text { Checksum } & -(16+\text { blocksize }) & \text { integer } \\ \text { Data block } & \text { clinic name index } & \text { word } \\ \text { investigator name index } & \text { word } \\ & \text { street index } & \text { word } \\ \text { streetnumber index } & \text { word } \\ \text { city index } & \text { word } \\ \text { country index } & \text { word } \\ & \text { postcode index } & \text { word } \\ \text { phone index } & \text { word } \\ \text { fax index } & \text { word } \\ \text { comments index } & \text { word } \\ \text { clinic name } & \text { string [N] } \\ \text { investigator name } & \text { string [M] } \\ \text { street } & \text { string [O] } \\ \text { streetnumber } & \text { string [P] } \\ \text { city } & \text { string [Q] } \\ \text { country } & \text { string [R] } \\ \text { postcode } & \text { string [S] } \\ \text { phone } & \text { string [T] } \\ \text { fax } & \text { string [U] } \\ \text { comments } & \text { string [V] }\end{array}$

The phone and fax fields should include the area code as well as the local number.

An empty string may be stored in any of the string fields for which information is not available. In this case the length $(N, M$, etc.) of the string is 1.

Pressure-Flow Studies of Voiding, Urethral Resistance and Urethral Obstruction Terminology 301

The index fields contain the position (in bytes) of the corresponding text field, relative to the start of the data block. All the index fields must be completed.

Record Type 17: ICS Volume Record

This record contains the filling volume and the residual volume.

$\begin{array}{lll}\text { Record type } & 17 & \text { integer } \\ \text { Blocksize } & 4 & \text { integer } \\ \text { Checksum } & -(17+\text { blocksize }) & \text { integer } \\ \text { Data block } & \text { filling volume } & \text { integer } \\ & \text { residual volume } & \text { integer }\end{array}$

The following information should be stored:

In the field: 
filling volume the calculated volume in the bladder at the beginning of the pressure-flow study (in ml) residual volume the volume in the bladder at the end of the study, either calculated or measured directly (in $\mathrm{ml}$ ); if the calculated volume is negative the value zero should be stored

Record Type 18: The ICS Signal Value Record

The ICS signal value record contains the actual data samples for one of the urodynamic signals. It includes a record number, allowing a signal that is too long to fit into one record to be divided so as to span several records. If the signal spans more than one record the number of the first record should be 1 and the following records should be numbered $2,3, \ldots$ If there is only one record its record number should be 0 .

$\begin{array}{lll}\text { Record type } & 18 & \text { integer } \\ \text { Blocksize } & 2+N^{*} 2 & \text { integer } \\ \text { Checksum } & -(18+\text { blocksize }) & \text { integer } \\ \text { Data block } & \text { signal ID } & \text { byte } \\ & \text { record number } & \text { byte } \\ & \text { data samples } & \text { word }[N]\end{array}$

The following information should be stored in these fields:

In the field:

signal ID As specified in Section A9: Addendum.

record number As described above.

datasamples The binary samples themselves, 1 word $=2$ bytes $=1$ sample, stored in the order low byte, high byte.

Record Type 19: The ICS Measurement Description Record

This record indicates that the file is an ICS standard file and describes the measurement's start date and time, the number of signals stored and the number of records in the file.

\begin{tabular}{|c|c|c|c|}
\hline $\begin{array}{l}\text { Record type } \\
\text { Blocksize } \\
\text { Checksum } \\
\text { Data block }\end{array}$ & $\begin{array}{l}19 \\
1+\mathrm{N} \\
-(19 \\
\text { Meas } \\
\text { Name } \\
\text { Start }\end{array}$ & $\begin{array}{l}\text { minute } \\
\text { hour } \\
\text { day } \\
\text { month } \\
\text { year } \\
\text { ignals }\end{array}$ & $\begin{array}{l}\text { integer } \\
\text { integer } \\
\text { integer } \\
\text { byte } \\
\text { string }[N] \\
\quad \text { byte } \\
\text { byte } \\
\text { byte } \\
\text { byte } \\
\text { byte* } \\
\text { byte }\end{array}$ \\
\hline
\end{tabular}




\section{Number of records $\quad$ word}

${ }^{*}$ Year is expressed modulo 100; e.g. $1995=95,2005=05$. The following information should be stored in these fields:

In the field:

measurement type the version number of this document, multiplied by 10 (e.g., for this version 70 ). (Note that the version number may not contain hundredths or smaller decimal fractions.)

name the string "ICS standard pressure-flow study"

start the starting time and date of the measurement (if any of these are unavailable, the default value Hex:00 should be stored in the corresponding position: minute, hour, day, month and/or year)

number of signals the number of signals stored in this file (e.g., 3 if just intravesical pressure, abdominal pressure and flow rate are stored).

number of records the total number of records stored in this file (including this measurement description record)

\section{A6. Signals and Information to be Stored: Minimal Specification and Optional Extensions}

Minimally the following 3 signals should be stored in 3 records of type 18:

intravesical pressure

abdominal pressure

flow rate

For each signal certain associated information should be stored in a record of type 14 .

Optionally the following signals can also be stored in records of type 18, with associated information in records of type 14 :

EMG envelope

voided volume

The voided volume signal may be useful if this is the signal measured by the urodynamic system, and the flow rate signal is derived from it.

In addition to the signals, further information about the patient and the measurements should be stored in records of types 16, 17 and 19. Full demographic data for the patient can be stored in a record of type 15 . Optionally a free format comment can be stored in a record of type 9 , and if detailed comments relating to events during the measurement are available they can be stored in records of type 8 .

\section{A7. Typical FileStructure}


Of the various record types, some are mandatory and some are optional. The structure of the file describing a particular measurement varies according to what records are stored.

The order of the records within the file is arbitrary in principle. However, it may be convenient to place records containing easily recognisable strings near the beginning, so that the file can quickly be identified if it is accidentally renamed or misplaced. In particular, record type 19 should preferably be the first one.

Thus a typical file structure might be:

Record type 19: ICS measurement description record (identifies file as an ICS standard file)

Record type 16: ICS source record (identifies originating clinic and investigator) Record type 9: Measurement comment record (identifies patient and type of measurement) Optionally, record type 15: ICS patient data record (contains full demographic data for patient)

Record type 14: ICS signal property record (describes properties of intravesical pressure signal)

Record type 14: ICS signal property record (describes properties of abdominal pressure signal)

Record type 14: ICS signal property record (describes properties of flow rate signal)

Optionally, further records of type 14 for voided volume and EMG envelope Record type 18: ICS signal value record (contains actual intravesical pressure data)

Record type 18: ICS signal value record (contains actual abdominal pressure data)

Record type 18: ICS signal value record (contains actual flow rate data)

Optionally, further records of type 18 for voided volume and EMG envelope

Optionally, record type 8: measurement marker record (identifies position and type of marker in data, the signal with which it is associated and a comment)

Optionally, further records of type 8 with additional markers

Record type 17: ICS volume record (contains filling volume prior to pressure-flow study and residual urine volume after voiding)

\section{A8. Acknowledgements}

This draft proposal is based partly on the ICS Measure File Format (ICSMFF) version 1.00 of 14.10.93, which was written by Michael Gondy Jensen, formerly of the Wiest company and now of Andromeda Medical Systems, and also on an earlier proposal for a simple digital pressure-flow standard circulated by Ron van Mastrigt. The Dantec, Laborie, Life-Tech and 
Wiest companies have agreed in principle to support a standard similar to the ICSMFF. The Life-Tech company has agreed to support the approved ICS standard.

\section{A9. Addendum:Signal IDs}

In records of types 8,14 and 18 , a signal ID of the type byte is specified. Corresponding signal names and units of the type string are required in record type 14. The following signal ID values, signal names and units have been defined:

$\begin{array}{llll}\text { For intravesical pressure } & \text { Signal ID } & \begin{array}{l}\text { Signal name } \\ \text { pves }\end{array} & \begin{array}{l}\text { Unit } \\ \mathrm{cmH}_{2} \mathrm{O}\end{array} \\ \text { abdominal pressure } & 1 & \text { pabd } & \mathrm{cmH}_{2} \mathrm{O} \\ \text { flow rate } & 2 & Q & \mathrm{ml} / \mathrm{s} \\ \text { EMGenvelope } & 3 & \mathrm{EMG}_{\text {env }} & \mathrm{uV} \\ \text { voided volume } & 4 & V_{\text {void }} & \mathrm{ml}\end{array}$

The following are reserved for possible future use:

$\begin{array}{llll} & \text { Signal ID } & \text { Signal name } & \text { Unit } \\ \text { For } P_{\text {det }} \text { acquired independently } & 6 & P_{\text {det }} & \mathrm{cmH}_{2} \mathrm{O} \\ \text { infused volume } & 7 & V_{\text {inf }} & \mathrm{ml} \\ \text { directEMGsignal } & 8 & \text { EMG } & \mathrm{uV} \\ \text { urethral pressure } & 9 & P_{\text {ura }} & \mathrm{cmH}_{2} \mathrm{O} \\ \text { urethral closure pressure } & 10 & P_{\text {clos }} & \mathrm{cmH}_{2} \mathrm{O}\end{array}$

Additional signal IDs, signal names and units may be introduced; proposed additions should be communicated to the ICS for registration. 